\title{
Logical Dimensions for the Information Provided by a Virtual Guide
}

\author{
Luisa Marucci, Fabio Patern $\square$ \\ CNUCE-C.N.R., Via V.Alfieri 1, Ghezzano-Pisa, Italy \\ \{luisa.marucci, fabio.paterno\}@cnuce.cnr.it
}

\begin{abstract}
If we consider most current applications we can notice a lack of support able to adapt to the different information needs that different users may have regarding a certain topic. In this paper, we present a solution that integrates a virtual assistant, able to implement adaptive support, in an adaptable application and discuss the logical dimensions of the information that can be provided by the virtual guide.
\end{abstract}

\section{Introduction}

Adaptive interfaces are flexible because can react in a wide set of modalities depending on the user interactions. This gives adaptive interfaces the possibility of supporting the needs of users in a more tailored way. However, there are two main risks. Wrong deductions can be inferred so that the tailored reaction does not match the user interest or the user does not understand the reasons of the change of behaviour and has a sense of disorientation and frustration. Thus, when designing adaptive support it is important to allow users to clearly understand: when the adaptive support can be activated; how the adaptive support provides information; which criteria determine the generation of information provided by the adaptive support. An application area where adaptive techniques can be particularly suitable is the museum field. A few research prototypes have been developed to use adaptive support in museum applications. This is the application domain where we have applied our approach.

We aim to design an environment where the adaptivity can be easily understood by users and enrich and facilitate navigation through the available information. To this end, users must have full control over when activating adaptive navigation. Then, users should be supported, during their visit, by an agent using a number of techniques. More precisely, we want the information delivered to be adaptive according several logical dimensions [3]:

- Introduction information, whenever a new topic or aspect is accessed the agent should provide introduction information on that topic;

- Summary information, the system should be able to provide some summary information concerning the items that have been accessed in the current session;

- Comparison information, where the purpose is to compare the current information with that previously accessed for some common aspect;

P. Brusilovsky, O. Stock, C. Strapparava (Eds.): AH 2000, LNCS 1892, pp. 359-362, 2000.

(c) Springer-Verlag Berlin Heidelberg 2000 
- Difference information, in this case the purpose is to indicate an attribute that was not present in the previous information;

- Curiosity information, indicating related information that can raise the interest of the user.

Thus, we aim to obtain a wide set of logical dimensions to discuss and present information taking into account also previous works such as that in [1] that focuses on an articulated set of comparisons (illustrative, clarificatory and direct). We have used this approach to design a Web application where there are two main components in the user interface: one part is dedicated to provide the information on works of art in an adaptable manner (the user selects at the beginning one user model and this determines the type of information presented); in the other part, there is the adaptive support that is provided through a virtual assistant that supplements the information presented with additional information belonging to the categories previously introduced. The adaptive support takes into account the current user model because different types of users can be interested in different types of additional information and different ways to group the information that is analysed.

\section{The Application}

The adaptive support has been designed for an application we developed beforehand, an adaptable virtual museum [2] that was designed following a model-based approach. The original application supported three user models (tourist, student, expert). At the beginning of the session, the user has to select one of them. According to the user model selected, the application provides different support mainly in three aspects: access to the information available, presentation of the information selected, modality of navigation.

To give full control to users on the adaptive support, when the initial choice of the user model appears we have added the possibility of selecting how adaptive support should be activated. Users can choose among three options: activation of the virtual guide, keeping disabled the virtual guide, possibility of activating the virtual guide during the navigation. If the last option is selected, when a work of art is presented there is also an additional button that allows the user to activate the virtual guide at any time.

When the agent is activated, beside the presentation of a work of art, there is a part of the main window dedicated to the comments of the virtual guide. The additional information provided through the virtual guide aims to make the users $\square$ visit more interesting and pleasant. Another goal is to provide additional dynamic information that help users link the standard information associated with each work of art, in a manner similar to when a visitor is accompanied by a real museum guide. Thus, at any time we have both standard information that is provided in any case associated with the work of art selected, and the agent-based support that provides additional information taking into account the user model and the interactions performed. 


\section{The Logical Dimensions of the Information Provided}

The virtual guide provides the types of information introduced before with a content tailored for the application considered:

Introduction information. When the user accesses some new aspect (for example a new artist or a new section) then the system provides information useful to introduce it. For example, when the user accesses the work of a new artist, the system provides some information on that artist taking into account the current user model and the works previously accessed. In the case of the tourist user model, the introduction of a new artist highlights how many works of that artist are contained in the museum and where they are located, specifying whether in a single or multiple rooms and the name of such rooms. When the student user model is active, the system generates introductions mainly in terms of definitions, thus helping users to create the association work-definition. Whenever a student accesses a new definition the system provides general concepts, describing materials used to process that definition, the artists that worked for that definition and the historical periods when it is possible to find them. In the case of an expert user model the introduction of a new artist has to take into account that the user is interested in a detailed search among the information contained in the museum, so it is preferable giving additional information concerning chronological motivations, observations, critiques, and historical notions.

Summary information. After having displayed a number of works of art, it can be useful to provide the user with a summary of the most important aspects that have been considered, supplemented by some related information. For example, if the user visits several works from the same historical period this can be interpreted as a strong interest in works belonging to that period. Then, a summary of the most important aspects of that historical period can be provided. The purpose of this information is to highlight the most important aspects common to the works of arts visited. The summary should help a further assimilation of notions already exposed to the user. Summary information is available after a certain number of accesses to the system and it depends on the current user model. Thus, for the tourist the summary will be related to the museum rooms visited whereas in the case of expert and student users will be related to the historic period considered. Additional links to the list of works visited can accompany the summary.

Comparison Information. Comparison is a good tool for supporting learning and can be easily memorised by the user. It allows users to relate works of art or compare them, for example comparing dimensions, chronology. There are different ways to connect a work to those previously seen. More specifically, for every current user model we want to consider aspects concerning artist, historical period, and material used. When there is a change of artist, before comparing two artists, the system controls what are the topics that the user is familiar with considering the works previously accessed and the current user model. Based on this analysis, the system decides whether to apply the comparison, if there are aspects to consider in comparing the two artists, which parameters to use for the comparison. If the comparison between the two artists has already occurred in the session then it is not repeated. 
Regarding the aspects to consider in the comparison, the system has to identify the main features of the artist (techniques used, preferred material, $\square$ ) by analysing the information contained in the application. The access to a new artist can occur along with the access to a new material, historical period, or definition. Thus, the additional comments have to consider all the changes occurred. If the user goes often back to works previously visited, this is interpreted as a strong interest for comparison information and thus this type of information is provided more frequently.

Difference information. In this case, the purpose is to highlight the difference between a work and those previously accessed. This information is useful for the user to better learn and remember the descriptions of the works of art. It can be triggered for two types of reasons: after the user has accessed some works then, when there is a work of art that completely differs for some aspect from all those previously visited, this is reported by the virtual assistant. For example, when there is a work of art made with a material that was not used for all the previously visited works. Otherwise, when there are works of arts that differ for some aspects that some virtual visitors may not catch then the difference is highlighted. For example, if after having accessed a high-relief the user selects a bas-relief then the virtual guide indicates the difference between these two techniques in order to avoid misunderstanding that can occur as they produce results that are visually similar.

Additional curiosity information. They are additional peculiar information that can increase the involvement of the visitor highlighting the features of the work that can raise the user $s$ interest. This type of information is generated whenever a work that is unique under some aspect is accessed. For example, if the current work of art was made with a material that has not been used for any other work included in the application then the system highlights this feature.

To conclude, we have introduced a solution supporting an integration of a virtual assistant with adaptive behaviour in a previously adaptable application. The adaptive support has been designed following a set of clearly understandable criteria and the information provided makes the users $\square$ visits more pleasant, increases their involvement, and better matches their interests.

\section{References}

1. M. Milosavljevic, Augmenting the User"s Knowledge via Comparison, Proceedings of UM 97 , Springer Verlag.

2. F. Patern $\square$ C. Mancini, Developing Adaptable Hypermedia, Proceedings Intelligent User Interfaces 99 , pp. 163-170, ACM Press.

3. F. Patern $\square$ Cristiano Mancini, Effective Levels of Adaptation to Different Types of Users in Interactive Museum Systems, Journal of the American Society for Information Science, Vol.51, N.1, pp.5-13, Wiley, 2000. 